\title{
Detection of RET Mutations in Multiple Endocrine Neoplasia Type 2a and Familial Medullary Thyroid Carcinoma by Denaturing Gradient Gel Electrophoresis
}

\author{
Michael L. Peacock, Marilyn J. Borst, Jason D. Sweet, and Ruth A. Decker* \\ Department of Surgery, University of Michigan Medical Center, Ann Arbor, Michigan 48109; Fax: 313-763-7307 \\ Communicated by Kenneth K. Kidd
}

\begin{abstract}
Germline missense mutations within the coding region of the RET proto-oncogene have recently been described in patients with the dominantly inherited cancer syndromes, multiple endocrine neoplasia type 2a (MEN 2a) and familial medullary thyroid carcinoma (FMTC). To date, the sequence variations occur in RET exons 10 and 11 and alter highly conserved cysteine residues in the proposed extracellular domain at codons $609,611,618,620$, and 634 . To expedite rapid screening of populations at risk of MEN 2a or FMTC, we developed a PCR-based denaturing gradient gel electrophoresis (DGGE) strategy that detects polymorphisms occurring at all five Cys codons in both RET exons using identical gel conditions. In this report, the screening results from DGGE analysis of 15 distinct MEN 2a and FMTC mutations ate shown. Each mutation generated a clearly distinguishable and unique homo- and heteroduplex band pattern. Given the highly efficient, reproducible, and sensitive nature of this approach, DGGE is particularly appropriate for rapid, large-scale screening of patients. Since prior knowledge of the RET mutation is unnecessary for analysis, DGGE is potentially valuable for distinguishing germline from seemingly sporadic medullary thyroid cancer as well as identifying novel sequence changes. 1996 Wiley-Liss, Inc.
\end{abstract}

KEY woRDs: MEN 2a, FMTC, DGGE, RET proto-oncogene

\section{INTRODUCTION}

Multiple endocrine neoplasia type 2a (MEN 2A) and familial medullary thyroid carcinoma (FMTC) are dominantly inherited cancer syndromes characterized by the occurrence of medullary thyroid carcinoma (MTC). Patients with MEN 2A also have a predisposition to the development of pheochromocytomas, parathyroid hyperplasia, and Hirschsprung's disease (Decker, 1992; Borst et al., 1995). Recently, germline missense mutations within the coding region of the RET proto-oncogene, a putative transmembrane tyrosine kinase, have been described in family members affected with these cancer syndromes (Mulligan et al., 1993; Donis-Keller et al., 1993; Mulligan et al., 1994). To date, all reported mutations occur in RET exons 10 and 11 and involve codons specifying highly conserved cysteine residues within the presumed extracellular domain of the receptor. Four of the five cysteines mutated in these syndromes are located in RET exon 10 at codons $609,611,618$, and 620 (Takahashi et al., 1988; Takahashi et al., 1989). The most frequent events occur at the remaining codon 634 in exon 11 , accounting for approximately $84 \%$ of patients with MEN 2a and $50 \%$ of patients with FMTC (Mulligan et al., 1994).

Identification of the responsible gene allowed us to develop a DNA-based screening strategy for mutation detection to assist in the diagnosis of patients at risk for MEN 2A or FMTC. Previously, testing for hereditary MTC was accomplished by measuring calcitonin levels after the intravenous injection of pentagastrin and/or calcium (Wells et al., 1978). This method of diagnosis is suboptimal for several reasons. First, due to delayed biochem-

Received August 19, 1994; accepted January 6, 1995. tressed. 
ical penetrance, years of repeated testing may be required before the disease can be safely excluded. Furthermore, borderline results are difficult to interpret and family compliance with screening problematic given the expensive and unpleasant nature of the pentagastrin testing. Finally, biochemical testing of all first-degree relatives in new cases of MTC to distinguish germline from sporadic disease is cumbersome and difficult to accomplish. More recently, various DNA-based techniques have emerged to identify RET mutations including direct genomic sequencing, single-stranded conformational polymorphisms (SSCP), and restriction enzyme digestion analysis (Donis-Keller et al., 1993; McMahon et al, 1994; Xue et al, 1994). However, these may not be the most efficient or accurate methods for mutational analysis. Moreover, prior knowledge of the mutation may be necessary limiting test efficacy in prospective screening. Therefore, we have developed a highly reliable denaturing gradient gel electrophoresis (DGGE) strategy to expedite detection of the MEN 2a/FMTC RET point mutations in exons 10 and 11. Analysis by DGGE has been successful in our hands as a nonradioactive method for rapid, simultaneous screening of a large number of patients and has replaced other DNA-based approaches to diagnosis as well as biochemical testing in our patients at risk of hereditary MTC.

\section{MATERIALS AND METHODS Polymerase Chain Reaction}

Genomic DNA representing 15 distinct mutations at Cys codons 609, 611,618, 620, and 634 were analyzed. Polymerase chain reaction (PCR) amplification of RET exon 10 was performed using $1 \mu \mathrm{g}$ genomic DNA template, $2.2 \mu \mathrm{M}$ each oligonucleotide primer, $200 \mu \mathrm{M}$ each dNTP, $1.25 \mathrm{U}$ Taq DNA polymerase (Gibco BRL, Gaithersburg, MD), $10 \mathrm{mM}$ Tris (pH 8.3), $50 \mathrm{mM} \mathrm{KCl}, 2.0 \mathrm{mM}$ $\mathrm{MgCl}_{2}$ in a total volume of $50 \mu \mathrm{l}$. Conditions for amplification consisted of 35 cycles at $95^{\circ} \mathrm{C}$ for 1 min (denaturation), $72^{\circ} \mathrm{C}$ for $1 \mathrm{~min}$ (annealing and extension), followed by a single denaturing and annealing step at $100^{\circ} \mathrm{C}$ for $10 \mathrm{~min}$ using an automated thermocycler (MJ Research, Watertown, MA). Expected product size is $216 \mathrm{bp}$. The following primers were chosen from intronic sequences flanking the intron/exon boundaries of exon 10 after a melting profile analysis by Melt 87 (Lerman and Silverstein 1987), and included a GC-clamp: ${ }^{\prime}$ GCGCCCCCCGCCCCCGCCCCGCCCGCCGCGGCGCCCCAGGAGGCTG-

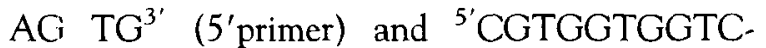
CCGGCCGCC $^{3 \prime}$ (3'primer).

For amplification of exon 11 , primers ${ }^{5} \mathrm{C}$ CTCACACCACCCCCACCCA ${ }^{3 \prime}$ (5'primer) and ${ }^{\prime}(\mathrm{GC})_{15}$ GTCCAGCGAGGGCCGGCG$\mathrm{GG}^{3^{\prime}}$ (3' primer) were selected from sequences flanking Cys codon 634 following MELT87 analysis. Amplification of the $268 \mathrm{bp}$ fragment was performed using $1 \mu \mathrm{g}$ genomic DNA, $2.2 \mu \mathrm{M}$ each primer, $200 \mu \mathrm{M}$ each dNTP, 1.25 units Taq polymerase, $10 \mathrm{mM}$ Tris (pH 8.3), $50 \mathrm{mM} \mathrm{KCl}, 1.5$ $\mathrm{mM} \mathrm{MgCl} 2$ and $10 \%$ DMSO in a final volume of $50 \mu \mathrm{l}$. Thermal cycling conditions consisted of 35 cycles of denaturation at $95^{\circ} \mathrm{C}$ for $1 \mathrm{~min}$, annealing at $62^{\circ} \mathrm{C}$ for $30 \mathrm{sec}$, and extension at $72^{\circ} \mathrm{C}$ for $1 \mathrm{~min}$ (MJ Research, Watertown, MD). Following each amplification, samples were heated to $100^{\circ} \mathrm{C}$ for 10 min and allowed to cool to room temperature to facilitate heteroduplex formation.

\section{Denaturing Gradient Gel Electrophoresis}

DGGE analysis was carried out on a 1 -mm-thick 9\% acrylamide gel (acrylamide:bis-acrylamide $20: 1)$ with a $50-80 \%$ linear gradient of denaturants $(100 \%=7 \mathrm{M}$ urea, $40 \%(\mathrm{v} / \mathrm{v})$ formamide $)$ in $1 \times$ TAE buffer $(40 \mathrm{mM}$ Tris acetate, $20 \mathrm{mM}$ sodium acetate, $1 \mathrm{mM}$ EDTA at $\mathrm{pH} 7.8$ ). Gel was overlayed with a $6 \%$ polyacrylamide stacker (acrylamide:bis-acrylamide 30:1) containing $1 \times \mathrm{TAE}$. Amplified PCR fragments were electrophoresed at $65^{\circ} \mathrm{C}$ and $17 \mathrm{~mA} /$ gel for $18 \mathrm{hr}$ (Hoefer Scientific Instruments, San Francisco, CA) with recirculation of buffer from the lower to upper electrode chamber and ethidium bromide stained (10 $\mathrm{min})$ prior to visualization.

\section{RESULTS}

Figure 1 illustrates the DGGE findings of 10 of the reported MEN 2a and FMTC RET mutations in exon 10. The analysis includes each of the four cysteine codons within the coding sequence of exon 10 implicated in the pathogenesis of MEN 2a and FMTC. The mutations shown occur at base positions 2021, 2028, 2047, 2048, 2053, and 2054 and involve Cys codons 609, 611, 618, and 620. Shown in Figure 2 are the results of DGGE analysis from five distinct Cys codon 634 mutations involving missense changes at nucleotides 2095, 2096, or 2097. In all cases, DGGE of the PCR-amplified fragment produced clear, sharply distinguishable and unique mutant homo- and heteroduplex band patterns specific for each point mutation. Normal controls showed the expected single banding pattern reflecting the homozygous genotype. Muta- 


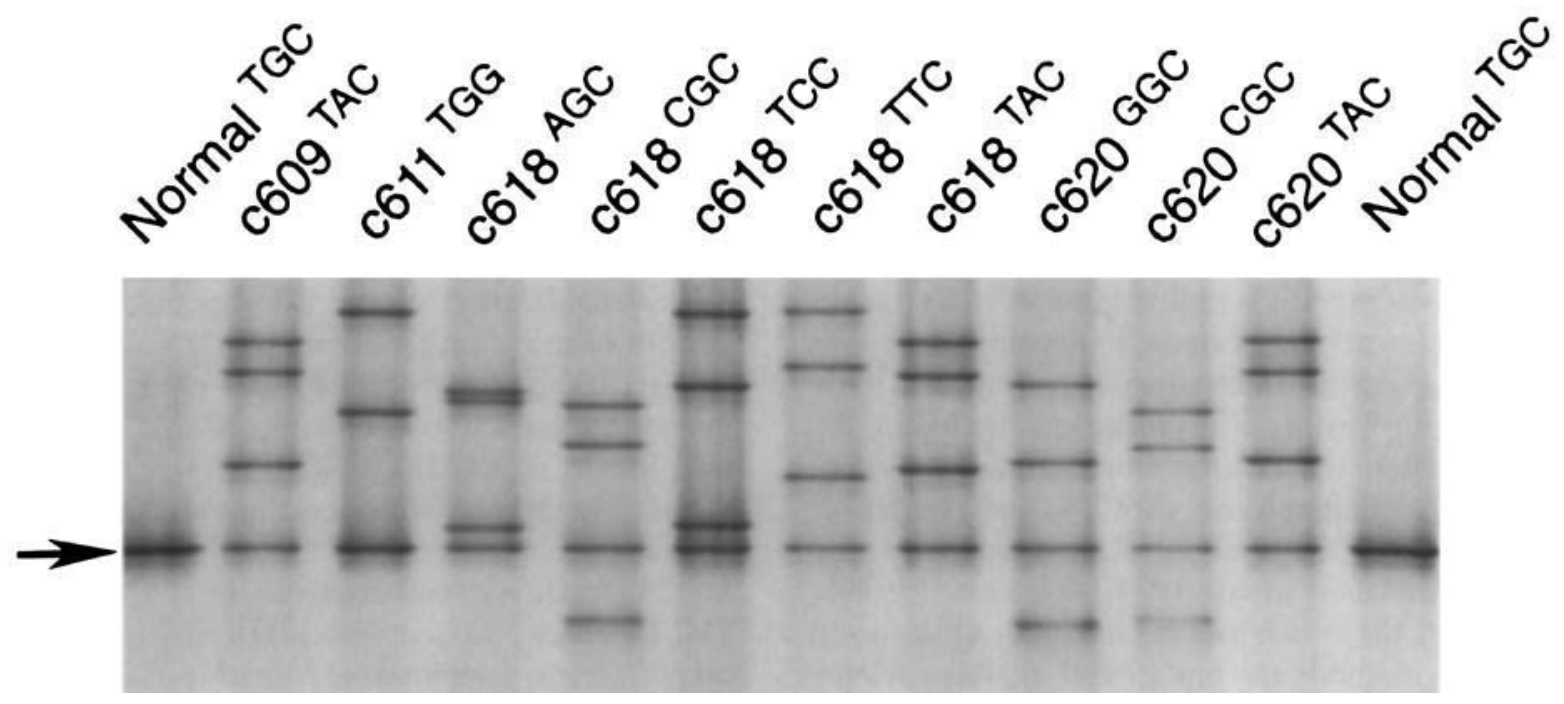

FIGURE 1. DGGE analysis of PCR amplified fragments representing 10 different missense mutations at Cys codons $609,611,618$, and 620 in exon 10 of the RET proto-oncogene and normal sequenced controls. Missense mutation and corresponding codon relative to the full length RET coding sequence are as indicated. Unique mutant and homo- and heteroduplex bands were observed in each case. Wild-type homoduplex is indicated by the arrow.

tions involving base pair transversions as well as transitions were successfully delineated. Normal and mutant homoduplex bands were superimposed in the TGC-to-TGG transversion at codons 611 and 634. Therefore, final classification of mutations in these cases relied on differences in the heteroduplex patterns. The DGGE results were consistently and easily reproducible in separate experiments under identical PCR and gel conditions.

The DGGE analysis of RET exon 10 in a segment of a large MEN 2A kindred is shown in Figure 3 . The mutant heteroduplex banding pattern is clearly present in all affected patients whereas the DGGE wild-type conformation, characterized by a single band, is seen in those unaffected with the disease. Direct DNA sequencing of mutants demonstrated a heterozygous base change of adenine for thymine at nucleotide 2047 resulting in a serine for cysteine substitution at codon 618 (not shown). All affected patients have undergone thyroidectomy with histological evidence of MTC. The normal status of individuals at risk for MTC in this family has been verified biochemically by the pentagastrin provocative testing.

\section{DISCUSSION}

The DNA samples analyzed collectively represent 15 of the reported missense mutations involving RET exons 10 and 11 in MEN 2A and FMTC. Our findings demonstrate that each point muta- tion reliably generated a DGGE polymorphism. Furthermore, DGGE of each mutation throughout this region of RET displayed a unique mutant homo- and heteroduplex banding pattern accurately reflecting the heterozygous point mutation. Final characterization of the mutations relied on direct comparison with known mutants and overall banding pattern. DGGE is a sensitive and highly reproducible technique that separates doublestranded DNA fragment under denaturing conditions based on differing melting characteristics (Fischer and Lerman, 1983). To ensure detection of base changes in the region of the lowest melting domain, a GC clamp was included in the sequence to be amplified. Given the high degree of resolution and reproducible nature of these results, we believe that all codon $609,611,618,620$, and 634 mutations can be identified, as well as novel mutations at other positions in patients with familial or seemingly sporadic MTC or pheochromocytoma.

Other techniques have been used to detect germline RET mutations including direct DNA sequencing, restriction enzyme digestion, and SSCP analysis, however, the DGGE strategy confers several advantages in this setting. First, because of the relatively few manipulations, this approach permits rapid turnover of results making it amenable to mass screening of patients. Second, it does not require the use of radioisotopes. Third, the proce- 

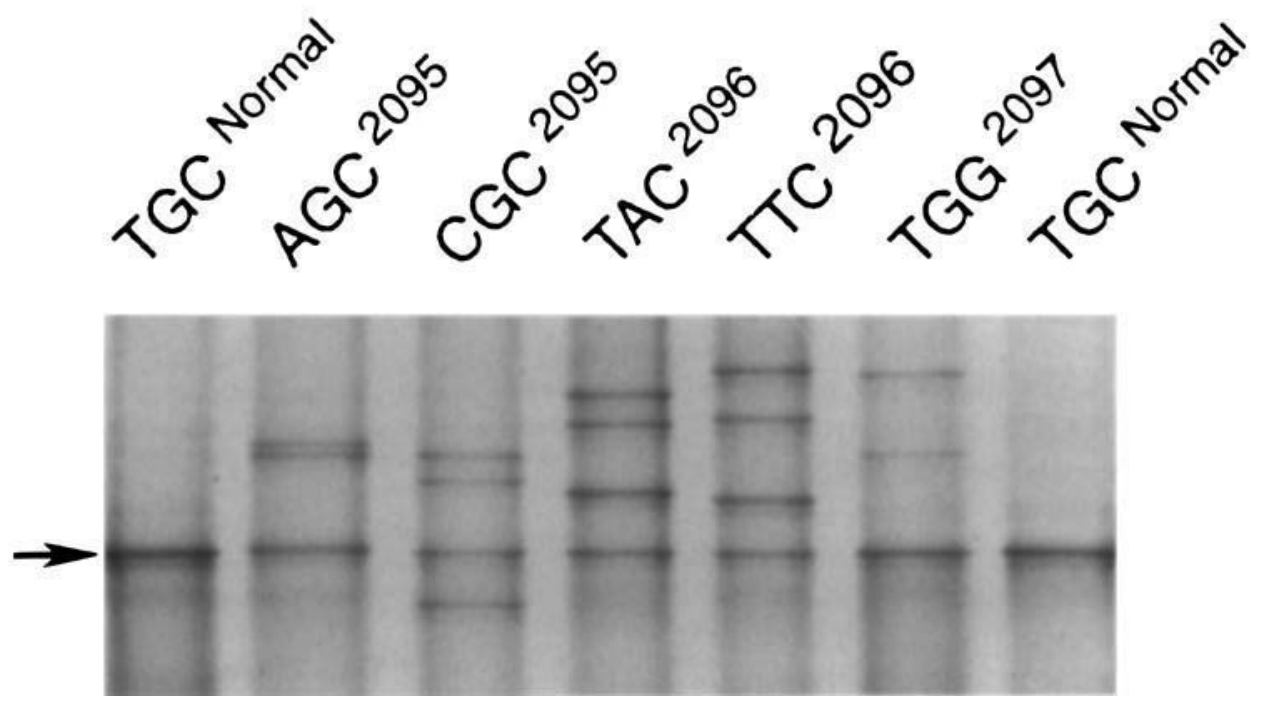

FIGURE 2. DGGE gel analysis of PCR-amplified fragments representing five different missense mutations at Cys codon 634 (nucleotides 2095, 2096, and 2097) in exon 11 of the RET proto-oncogene and normal sequenced and clinical controls. Missense mutation and corresponding basepair position relative to the RET coding sequence are as indicated. Nucleotides are numbered from the transcription start site as defined by Kwok et al. (1993) and Itoh et al. (1992). Unique mutant homo- and heteroduplex bands were observed in each case. Wild-type homoduplex is indicated by the arrow.
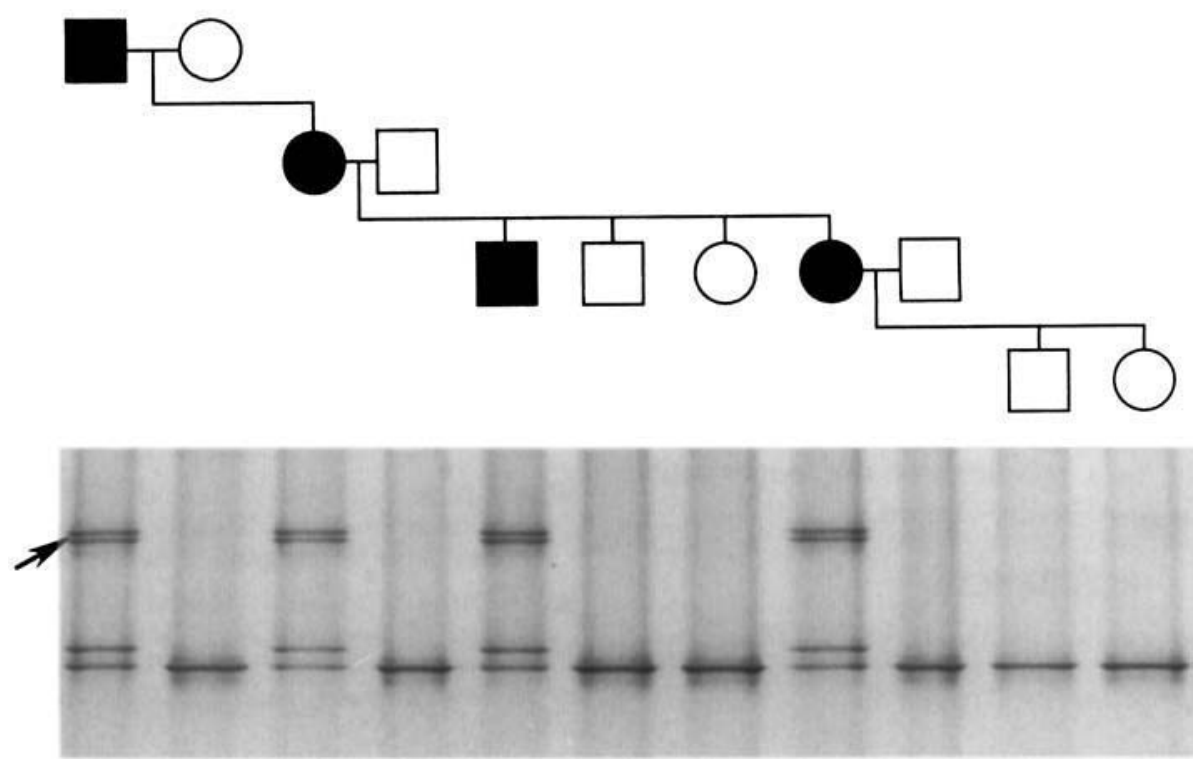

FIGURE 3. Pedigree and and DGGE gel analysis of RET Exon 10 from a family with MEN 2a. Direct DNA sequencing (not shown) revealed a thymine to adenine base substitution at nucleotide 2047 (Cys codon 618). Closed symbols indicate patients with MEN 2a. Unaffected individuals and spouses are indicated by the open symbols. All affected patients have histological evidence of MTC. Arrow, heteroduplexes generated from the mutant allele.

dure simultaneously tests for all possible mutations at each of the five codons using identical gel conditions making it highly efficient and cost-effective. The DGGE genotype can easily be identified in comparison with known mutant controls. Until greater experience is gained with our method, all mutations are confirmed by direct sequencing prior to diagnosis and treatment to ensure that the vari- 
ations observed are not benign polymorphisms. We anticipate that this method will readily identify novel RET germline mutations in patients with familial disease or seemingly sporadic MTC. It has been estimated that missense changes in one of these five cysteines accounts for $97 \%$ of mutations in patients with MEN 2a and $86 \%$ of those observed in FMTC (Mulligan et al., 1994). Therefore, the DGGE approach described will not encompass all possible MEN 2a/FMTC mutations. Optimization of the DGGE conditions for RET exon 13, another potential site in which FMTC mutations occur (Eng et al., 1995) is underway. We are currently testing the efficacy of the DGGE diagnostic strategy in our patients at risk for MEN $2 \mathrm{a}$ or FMTC in a prospective ongoing clinical study. To date, discordancy between the DGGE results and findings at thyroid surgery has not been found (Decker et al., 1995). Based on our experience, DGGE is a convenient, inexpensive, highly sensitive and reliable nonradioactive method which can be employed for rapid large-scale screening of patients at risk for RET MEN 2a and FMTC mutations.

\section{ACKNOWLEDGMENTS}

We are deeply grateful to Helen Donis-Keller, Samuel A. Wells, Jr. (Department of Surgery Washington University, St. Louis), Charis Eng, and Bruce A.J. Ponder (Department of Pathology, University of Cambridge, England) for DNA samples. This work was supported by grants to R.A.D. from the NIH (1K08DK02176-02), American Cancer Society (VM-73), and the John and Suzanne Munn Endowed Research Fund of the University of Michigan Comprehensive Cancer Center. R.A.D. is a recipient of the American College of Surgeons Faculty Award.

\section{REFERENCES}

Borst M, Van Camp J, Peacock M, Decker R (1995) Mutational analysis of multiple endocrine neoplasia type 2 a associated with Hischsprung's disease. Surgery 117:386-391.
Decker R (1992) Long-term follow-up of a large North American kindred with multiple endocrine neoplasia type 2a. Surgery 112:1066-1073.

Decker R, Peacock M, Borst M, Sweet J, Thompson N (1995) Progress in genetic screening of MEN 2a: Is calcitonin testing obsolete? Surgery 118:257-264.

Donis-Keller H, Dou S, Chi D, Carlson K, Toshima K, Lairmore T, Howe J, Moley J, Goodfellow P, Wells S (1993) Mutations in the RET proto-oncogene are associated with MEN 2a and FMTC. Hum Mol Genet 2:851-856.

Eng C, Smith D, Mulligan L, Healey C, Zvelebil M, Stonehouse T, Ponder M, Jackson C, Waterfield M, Ponder B (1995) A novel point mutation in the tyrosine kinase domain of the RET proto-oncogene in sporadic medullary thyroid carcinoma and in a family with FMTC. Oncogene 10:509-513.

Fischer SG, Lerman LS (1983) DNA fragments differing by single base-pair substitutions are separated in denaturing gradient gels: Correspondence with melting theory. Proc Natl Acad Sci USA 80:1579-1583.

Itoh F, Ishizaka Y, Tahira T, Yamamoto M, Miya A, Imai K, Yachi A, Takai S, Sugimura T, Nagao M (1992) Identification and analysis of theret proto-oncogene promoter region in neuroblastoma cell lines and medullary thyroid carcinomas from MEN 2A patients. Oncogene 7:1201-1206.

Kwok J, Gardner E, Warner J, Ponder B, Mulligan L (1993) Struc tural analysis of the human RET proto-oncogene using exon trapping. Oncogene 8:2575-2582.

Lerman L, Silverstein K (198\%) Computational simulation of DNA melting and its application to denaturing gradient gel electrophoresis. Methods Enzymol 155:482-501.

McMahon R, Mulligan L, Healey C, Payne S, Ponder M, Ferguson-Smith M, Barton D, Ponder B (1994) Direct, non-radioactive detection of mutations in Multiple Endocrine Neoplasia Type 2a families. Hum Mol Genet 3:643-646.

Mulligan L, Kwok J, Healey C, Elsdon M, Eng C, Gardner E, Love D, Mole S, Moore ], Papi L, Ponder M, Telenius H, Tunnacliffe A, Ponder B (1993) Germ-line mutations of the RET proto-oncogene in multiple endocrine neoplasia type $2 \mathrm{a}$. $\mathrm{Na}$ ture 363:458-460.

Mulligan L, Eng C, Healey C, Clayton D, Kwok J, Gardner E, Ponder M, Frilling A, Jackson C, Lehnert $H$, Neumann $H$, Thobodeau S, Ponder B (1994) Specific mutations of the RET proto-oncogene are related to disease phenotype in MEN 2a and FMTC. Nature Cienet 6:70-74.

Wells S, Baylin S, Linehan W, Farrell R, Cox E, Cooper C (1978) Provocative agents and the diagnosis of medullary thyroid carcinoma of the thyroid gland. Ann Surg 188:139-141.

Xue F, Hong Y, Maurer L, Memoli V, Nutile-McMenemy N, Schuster M, Bowden D, Mao J, Noll W (1994) Germline RET mutations in MEN 2A and FMTC and their detection by simple DNA diagnostic tests. Hum Mol Genet 3:635-638. 